\title{
Nota Del DiRetTore
}

Questo nuovo numero di Quaderni d'italianistica segna una svolta nella vita della rivista: dopo quattro anni, il testimone passa a un nuovo comitato editoriale. Nell'assumere la direzione, vorrei prima di tutto ringraziare il mio predecessore, Gianni Cicali, per lo straordinario impegno con cui ha svolto il suo mandato. Quaderni gode di una solidissima reputazione internazionale, confermandosi una delle più importanti riviste di studi italiani in Nord America, e questo sicuramente grazie all'abilità con cui il Prof. Cicali ha saputo coordinare il lavoro di un grande numero di specialisti - autori, recensori, curatori di numeri speciali, referees, ecc. - garantendo che ciascun fascicolo della rivista offrisse i migliori risultati della ricerca attuale nei campi della letteratura, del cinema, della linguistica, e della pedagogia della lingua. Un grande ringraziamento va poi anche al comitato editoriale che ha validamente assistito il Prof. Cicali, in particolare ai due co-direttori Patrizia Bettella e Konrad Eisenbichler, e ai membri del prestigioso comitato scientifico internazionale.

Se Quaderni d'italianistica riuscirà nei prossimi anni a mantenere gli alti standard di rigore scientifico e originalità della ricerca stabiliti dal mio predecessore, ciò sarà dovuto in massima parte al fatto che ho la fortuna di poter contare su un ottimo comitato editoriale che rappresenta bene la diversità dell'italianistica canadese: i due direttori associati Elena Benelli e Franco Pierno, le responsabili delle recensioni Cristina Caracchini e Patrizia Bettella, la managing editor Sarah Rolfe Prodan, e Konrad Eisenbichler, che si occuperà della promozione della rivista. Li ringrazio in anticipo, insieme ai membri del nuovo comitato scientifico che hanno generosamente acconsentito a dare il loro patrocinio - e i loro consigli — alla rivista. Last ma naturalmente not least, ringrazio gli autori che vorranno offrire la loro collaborazione, che, ricordiamo, può essere in ciascuna delle tre lingue ufficiali della rivista: italiano, inglese o francese.

\section{Luca Somigli}

